\title{
GOLDEN 2-LIKE Transcription Factors of Plants
}

\author{
Min Chen ${ }^{1,2,3}$, Meiling Ji1,2,3, Binbin Wen ${ }^{1,2,3}$, Li Liu'1,2,3, Shaoxuan Li, 1,2,3, Xiude Chen ${ }^{1,2,3}$, \\ Dongsheng Gao ${ }^{1,2,3 *}$ and Ling $\mathrm{Li}^{1,2,3 *}$
}

${ }^{1}$ State Key Laboratory of Crop Biology, Shandong Agricultural University, Taian, China, ${ }^{2}$ College of Horticulture Science and Engineering, Shandong Agricultural University, Taian, China, ${ }^{3}$ Shandong Collaborative Innovation Center for Fruit and Vegetable Production with High Quality and Efficiency, Taian, China

Golden2-like (GLK) transcription factors are members of the GARP family of Myb transcription factors with an established relationship to chloroplast development in the plant kingdom. In the last century, Golden2 was proposed as a second golden producing factor and identified as controlling cellular differentiation in maize leaves. Then, GLKs were also found to play roles in disease defense and their function is conserved in regulating chloroplast development. Recently, research on GLKs has rapidly increased and shown that GLKs control chloroplast development in green and non-green tissues. Moreover, links between phytohormones and GLKs were verified. In this mini-review, we summarize the history, conservation, function, potential targets and degradation of GLKs.

Keywords: GLKs, chloroplast development, pathogen resistance, senescence, transcription factors

\section{INTRODUCTION}

In plants, the development of functional chloroplasts is dependent on tight co-ordination between chloroplast and nuclear genomes. Previous studies estimated that almost 3000 nuclear-encoded proteins are located in the chloroplast (Pedro et al., 2012). In the nuclear genome, Golden2-like $(G L K)$ transcription factors have been shown to be involved in the related expression of nuclear chloroplast-localized proteins and photosynthesis-related genes in maize (Zea mays), the moss Physcomitrella patens and Arabidopsis thaliana (Hall et al., 1998; Rossini et al., 2001; Fitter et al., 2002; Yasumura et al., 2005; Waters et al., 2009). The name, Golden2, follows nomenclature used for the first golden producing factor, golden1, found in maize by Jenkins (1926).

\section{HISTORY}

Golden2 was named in maize almost 90 years ago and subsequently researchers found many Golden2-like (GLK) genes in Arabidopsis, rice (Oryza sativa), moss (P. patens), pepper (Capsicum annuum) and tomato (Solanum lycopersicum; Fitter et al., 2002; Bravo-Garcia et al., 2009; Powell et al., 2012; Brand et al., 2014). Then, Golden2, with TEA DNA-binding domains, was shown to encode a $2.2-\mathrm{kb}$ transcript mainly in maize leaves. In addition, Golden 2 plays a critical role in cellular differentiation (Hall et al., 1998); and GLK expression in maize, rice and Arabidopsis is regulated by light (Rossini et al., 2001; Fitter et al., 2002), while GLKs in Brassica spp. are induced by cold stress (Savitch et al., 2005). Subsequently, Golden 2 was classified into the GARP family, named by Riechmann et al. (2000). In most characterized plant genomes, including Arabidopsis, 
maize, rice, sorghum (Sorghum bicolor), and P. patens (i.e., from bryophytes to angiosperms), GLK genes exist as pairs.

\section{EVOLUTION AND CONSERVATION OF GLKs IN DIVERSE PLANTS}

Land plants have 1-4 GLKs but no GLKs have been found in sequenced algal genomes (Wang et al., 2013). Wang et al. (2013) also demonstrated that the ancestral state of flowering plants was a single $G L K$ gene and that gene duplication occurred in specific species. P. patens contains two GLKs due to a recent genome duplication within this species (Yasumura et al., 2005; Rensing et al., 2008). In the $\mathrm{C}_{4}$ plants, maize, and sorghum, GLK1 and GLK2 are expressed in bundle sheath (BS) and mesophyll (M) cells, respectively, associated with chloroplast dimorphism. However, in Cleome gynandra, another $\mathrm{C}_{4}$ plant, CgGLK1 and CgGLK2 are both expressed in BS and M cells and expression is higher in $\mathrm{M}$ cells. Of the identified $\mathrm{C}_{4}$ plants, compartmentalization of GLK function is not necessary in the development of chloroplast (Wang et al., 2013). Although the function of GLKs is conserved, there has been specialization of the GLK pathway (Bravo-Garcia et al., 2009). During the evolution from $P$. patens to Arabidopsis, the upstream and downstream targets of GLKs may have diverged.

GLK genes contain two highly conserved domains: a C terminal GCT-box which is specific to GLK genes and a DNAbinding domain (DBD) at the C terminal (Rossini et al., 2001). The AREAEAA hexapeptide sequence at the DBD is highly conserved among the GARP family (Hosoda et al., 2002). This DBD occurs in green algae and land plants, whereas GCT-box is found only in land plants.

\section{FUNCTION}

\section{Cellular Differentiation}

In all $\mathrm{C}_{4}$ plants, there is differentiation of three photosynthetic cell-types in leaf blades: $\mathrm{C}_{4} \mathrm{BS}$ and $\mathrm{C}_{4}$ and $\mathrm{C}_{3} \mathrm{M}$ cells. In maize, G2 and ZmGlk1 transcripts accumulate primarily in $\mathrm{C}_{4}$ tissues. In the $\mathrm{C}_{3}$ model plant rice and Arabidopsis, the GLKs act redundantly in promoting photosynthetic development (Rossini et al., 2001; Fitter et al., 2002). Therefore, these studies provide support for the idea that GLKs not only control cell-type differentiation processes but also play crucial roles in chloroplast development (Riechmann et al., 2000; Rossini et al., 2001).

\section{Chloroplast Development}

GLKs play pivotal roles in regulating chloroplast development in diverse plant species. Although the function of GLKs is conserved, different genetic mechanisms may operate upstream and downstream of GLK function in diverse species. Chloroplast development is an essential process in all plant cells and three types of chloroplasts exist in $\mathrm{C}_{4}$ plants. In one of the earliest studies, the size of chloroplasts and the numbers of thylakoid lamellae in $g 2$ mutants were both smaller than in wild type (Langdale and Kidner, 1994).
GLKs are potent positive regulators of chloroplast development in the plant kingdom. It has been verified that GLK1 is expressed mainly in leaves, while GLK2 is predominant in fruit. In Arabidopsis, Atglk1 Atglk2 mutants have pale-green photosynthetic tissues, lower levels of LHCB6 (a light harvesting chlorophyll $\mathrm{a} / \mathrm{b}$ binding protein) transcripts and an earlier flowering phenotype. There is a similar pale silique phenotype for Atglk2 and Atglk1 Atglk2 mutants (Fitter et al., 2002). Overexpression of either AtGLK1 or AtGLK2 in the double mutants led to completely restored levels of leaf chlorophyll (Chl) and LHCB6 transcripts and the time to flowering in a cell-autonomous manner (Waters et al., 2008). Leister and Kleine (2016) identified genomes uncoupled (gun) mutants as GLK overexpressors. In addition, galactolipid-synthesis genes which affect biogenesis of thylakoid membranes are also regulated by GLKs during leaf development (Kobayashi et al., 2014). In tomato, both SlGLK1 and SlGLK2 are expressed in leaves, whereas only SlGLK2 is expressed in fruit and is predominantly expressed in the green shoulder of fruit of the $U$ phenotype. This green shoulder is lost in the uniform ripening (u) mutant which was bred for evenly ripened fruit. It was demonstrated that SlGLK2 influences photosynthesis and the chloroplast developmental gradient in immature fruit (Powell et al., 2012; Nguyen et al., 2014). This conclusion was further verified by a promoter expressed later in fruit development. Furthermore, SlGLK2 expression is partially controlled by light (Powell et al., 2012). Co-suppression of SlGLK1 only resulted in pale-green leaves but showed no notable differences in fruit, while co-suppression of SlGLK2 mimicked the $u$ mutant (Powell et al., 2012; Nguyen et al., 2014). Overexpression of SlGLK2 and SlGLK1 resulted in uniformly darker green unripe fruit and enhanced nutritional quality in ripe fruit (Powell et al., 2012; Cheng and Lai, 2013; Nguyen et al., 2014). However, overexpression of GLK1 and GLK2 do not affect overall ripening regulation according to RNA-seq analysis, showing that the GLKs have no impact on general ripening control systems. In pepper, the role of CaGLK2 in regulating fruit development, and CaGLK2 expression throughout the entire fruit, has been studied (Brand et al., 2014). Furthermore, the KNOTTED1-LIKE HOMEOBOX (KNOX) genes act upstream of SlGLK2, implying that a regulatory mechanism of chloroplast development exists in fruit (Nadakuduti et al., 2014). All these suggest that GLK1 and GLK2 have functional equivalence and are tissue-specific. Interestingly, the tissue-specificity of SlGLK1 was demonstrated relating to the different Histone 3 Lysine 4 trimethylation (H3K4me3) levels in the promoter region of SlGLK1, whereas the equivalent SlGLK2 locus was not detected (Nguyen et al., 2014).

Plant roots are generally non-green and heterotrophic organs. Roots of some epiphytic plants are green and perform active photosynthesis; however, in most cases, the plants still depend on aerial leaves for energy (Aschan and Pfanz, 2003). The auxin signaling pathway was shown to be involved in regulating chloroplast development through GLKs in fruit and also in roots (Kobayashi et al., 2012). The expressions of GLK1 and GLK2 are much lower in roots than in leaves (Fitter et al., 2002). Overexpression of GLKs not only results in derepressing chloroplast development but also triggers 
ectopic development of chloroplasts in roots. Although GLK overexpression (GLKOX) can enhance chloroplast development and induce chloroplast division, other plastids including amyloplasts were not affected in $G L K 1_{\mathrm{OX}}$ roots. Moreover, carbon dioxide fixation and phototrophic performance of GLKOX roots increased, implying that root photosynthesis may influence the effective carbon utilization in plants (Kobayashi et al., 2013). In $G L K 1_{\mathrm{OX}}$ and GLK2 $\mathrm{OX}$ roots, DGD1 was obviously upregulated together with $C H L H$, which both encode key enzymes of digalactosyldiacylglycerol synthesis. Kobayashi et al. (2014) also reported that genes involved in fatty acid desaturation were upregulated in $G L K 1_{\mathrm{OX}}$ roots.

\section{Biotic Stress}

The role of GLKs in disease defense is indicated in several studies of Arabidopsis (Savitch et al., 2007; Jhadeswar et al., 2014). The Arabidopsis genome contains two GLKs of the GARP family: AtGLK1 and AtGLK2. AtGLK1 Ox in Arabidopsis leads to significant up-regulation of genes related to the defense and salicylic acid (SA) signaling pathway, whereas, PR1 (an indicator of systemic acquired resistance activation) was down-regulated (Savitch et al., 2007). Compared with wild type, AtGLK1 $1_{\mathrm{OX}}$ plants exhibited stronger resistance to Fusarium graminearum and more susceptibility to the virulent oomycete pathogen Hyaloperonospora arabidopsidis ( $\mathrm{Hpa}$ ) Noco2 (Jhadeswar et al., 2014). Taken together, these observations confirmed that the AtGLK1 not only regulates disease defense-related genes, but also plays different roles when various pathogens challenge AtGLK1 $1_{\text {OX }}$ Arabidopsis. Following pretreatment with SA, a method used to induce resistance against Hpa Noco2 (Ryals et al., 1996; Kunkel and Brooks, 2002), 35S:AtGLK1 plants showed only marginally enhanced resistance to Hpa Noco2. The glk1 glk2 double-mutant displayed strong resistance to Hpa Noco2. This phenomenon was partially influenced by SA accumulation, and not mediated through NONEXPRESSOR OF PATHOGENESISRELATED 1 (Jhadeswar et al., 2014). Pretreatment with SA can marginally reduce resistance in $g l k 1$ glk2 plants. In all tested mutants, the SA signaling pathway is functional but does not provide resistance to Hpa Noco2. In addition, both the activation of the SA pathway and suppression of the jasmonic acid (JA) pathway are alternatives for resistance to Hpa Noco2 (Lawton et al., 1995; Clarke et al., 1998; Li et al., 2001; Murray et al., 2002; Rairdan and Delaney, 2002; Argueso et al., 2012; Massoud et al., 2012). Mutants pretreated with JA, the response of JA-treated mutants suggesting that the susceptible Arabidopsis to Hpa Noco2 requires integration of GLKs and JA signaling. However, AtGLK1-facilitated resistance to Botrytis cinerea is independent of JA signaling. In rice, OsGLK1 may play a role in resistance to pathogen invasion (Nakamura et al., 2009). Recent studies have indicated that AtGLKs also played a positive role in the tolerance to Cucumber mosaic virus (Han et al., 2016).

In addition, Nagatoshi et al. (2016) identified that GLKs affect ozone tolerance via controlling stomatal movement. The chimeric repressors for GLK1 and GLK2 (GLK1/2-SRDX) and $G L K 1 / 2$ overexpression (35S:GLK1/2) were used and the researchers found that GLK1/2-SRDX showed remarkable tolerance to ozone with no defects in $\mathrm{M}$ chloroplasts, while
35S:GLK1/2 was hypersensitive to ozone. In addition, GLKs also affect stomatal aperture and expression of genes controlling movement of stomata and of potassium ions.

\section{Senescence}

Previous studies have shown that both photosynthesis and Chl contents decline in senescent leaves. GLKs participate in the regulation of leaf senescence. It was identified that ATAF1 regulates senescence through GLK1 and ORE1. Rauf et al. (2013) verified that ORE1 and GLK proteins can specifically interact and form GLK1-ORE1 heteromers to repress expression of GLK target genes. Interestingly, micro164 is abundant in developing leaves and decreases during leaves senescence, and can repress ORE1 expression (Kim et al., 2009). Determining whether micro164 controls GLKs will require further study. GLK2 as the target gene of PIF4 (phytochrome-interacting factor 4) was significantly repressed by PIF4; and GLK1, a target gene of BZR1 and PIF4, was proposed to be regulated by them synergistically (Oh et al., 2012). Song et al. (2014) demonstrated that PIF4 could repress GLK2 expression and trigger senescence. Compared with wild type, 35S:GLK1 and 35S:GLK2 overexpressors showed delayed senescence, while glk1 and glk2 single mutants showed little change in their senescence behavior.

\section{GLKS AND PHYTOHORMONES}

Phytohormones play adjustment roles in plant growth and development. In several studies, phytohormones including auxin and brassinosteroids have been implicated in tomato fruit chloroplast development and correlated with increased SIGLK expression. In rice, OsGLK1 regulates chloroplast development, which is also controlled by light and phytohormones, and OsGLK1 plays roles in the late steps of Chl biosynthesis (Nakamura et al., 2009). Previous study identified that downregulation of DR12/SIARF4 in tomato may be related to the dark-green fruit phenotype (Jones et al., 2002). In SlARF4silenced lines, the expression of SlGLK1 and SlGLK2 is elevated, indicating that SlARF4 negatively regulates expression of SlGLK1 and SlGLK2 (Sagar et al., 2013; Nadakuduti et al., 2014). Several observations indicate that the relationship of DDB1 and GLK2 has an additive effect (Nguyen et al., 2014). The bes1-D seedlings are pale green and have a striking reduction in Chl. Subsequently, Yu et al. (2011) identified that BES1 acts to repress expression of $G L K 1$ and $G L K 2$, thereby adversely affecting chloroplast function. Whether BES1 controls GLK1 and GLK2 expression through PIL6 will be interesting to determine.

\section{POTENTIAL TARGETS OF GLKS}

Their primary target genes are light harvesting and Chl biosynthesis genes. GLKs directly bind to the promoters of target genes and act as transcriptional activators (Waters et al., 2009). GLKs interact with proline-rich regions of G-box-binding bZIP factors in Arabidopsis (Tamai et al., 2002). The G-box of light-regulated promoters is important for plastid retrograde signaling and GLKs are found to act downstream of the pathway. 
In addition, $\mathrm{K}^{+}$CHANNEL IN ARABIDOPSIS THALIANA1 (KAT1) may be a direct target of GLKs (Nagatoshi et al., 2016). Except their primary target genes are up-regulated in GLKsox, so does GLK-non-targeted genes associated with chloroplast development.

\section{UBIQUITIN-CONJUGATED DEGRADATION OF GLK TRANSCRIPTION FACTORS}

Levels of proteins such as SIGLK2 are negatively regulated by ubiquitin-proteasome system (UPS)-mediated proteolysis in eukaryotes (Tomko and Hochstrasser, 2012; Tang et al., 2015). In tomato, Tang et al. (2015) demonstrated that GLK2 associates with the CUL4-DDB1-DET1 E3 complex using co-immunoprecipitation and bimolecular fluorescence complementation. Two lysine residues (K11 and K253) of the GLK2 protein play an important role in the ubiquitin-mediated degradation of GLK2 (Tang et al., 2015).

\section{CONCLUSION AND PERSPECTIVES}

GLKs are members of the GARP family, conserved proteins that originated in bryophytes. Since the first isolation of G2 in 1927, GLKs have been identified in many plants. Their best described role is chloroplast development, although they also participate in disease defense and senescence. Summarizing the history,

\section{REFERENCES}

Argueso, C. T., Ferreira, F. J., Epple, P., To, J. P., Hutchison, C. E., Schaller, G. E., et al. (2012). Two-component elements mediate interactions between cytokinin and salicylic acid in plant immunity. PLoS Genet. 8:933-950. doi: 10.1371/journal.pgen.1002448

Aschan, G., and Pfanz, H. (2003). Non-foliar photosynthesis-a strategy of additional carbon acquisition. Flora 198, 81-97. doi: 10.1078/0367-2530-00080

Brand, A., Borovsky, Y., Hill, T., Rahman, K. A. A., Bellalou, A., Deynze, A. V., et al. (2014). CaGLK2 regulates natural variation of chlorophyll content and fruit color in pepper fruit. Theor. Appl. Genet. 127, 2139-2148. doi: 10.1007/s00122014-2367-y

Bravo-Garcia, A., Yasumura, Y., and Langdale, J. A. (2009). Specialization of the Golden2-like regulatory pathway during land plant evolution. New Phytol. 183, 133-141. doi: 10.1111/j.1469-8137.2009.02829.x

Cheng, L., and Lai, K. (2013). Golden2- like (GLK2) Transcription Factor: Developmental Control of Tomato Fruit Photosynthesis and Its Contribution to Ripe Fruit Characteristics. M.S. thesis, University of California, Davis, CA.

Clarke, J. D., Liu, Y., Klessig, D. F., and Dong, X. (1998). Uncoupling PR gene expression from NPR1 and bacterial resistance: characterization of the dominant Arabidopsis cpr6-1 mutant. Plant Cell 10, 557-569. doi: 10.1105/tpc.10.4.557

Fitter, D. W., Martin, D. J., Copley, M. J., Scotland, R. W., and Langdale, J. A. (2002). GLK gene pairs regulate chloroplast development in diverse plant species. Plant J. 31, 713-727. doi: 10.1046/j.1365-313X.2002.01390.x

Hall, L. N., Rossini, L., Cribb, L., and Langdale, J. A. (1998). Golden 2: a novel transcriptional regulator of cellular differentiation in the maize leaf. Plant Cell 10, 925-936. doi: 10.1105/tpc.10.6.925

Han, X. Y., Li, P. X., Zou, L. J., Tan, W. R., Zheng, T., Zhang, D. W., et al. (2016). GOLDEN2-LIKE transcription factors coordinate the tolerance to Cucumber conservation, function, and degradation of the GLK proteins, shows that little is known about the structure of GLKs or GLKs in perennial plants, and this area requires more study. Overall, $G L K s$, as part of chloroplast and nuclear genomes, control the development of chloroplasts, and determine the capacity for photosynthesis. Manipulating the expression patterns of GLKs may provide an opportunity to increase production and quality traits in many species.

\section{AUTHOR CONTRIBUTIONS}

MC, LL and DG designed the article. MC, MJ, BW, LiL, SL, $\mathrm{XC}$ wrote the manuscript and DG, LL revised the intellectual content of this manuscript. All authors read and approved the final manuscript.

\section{FUNDING}

This study was funded by the National Natural Science Foundation of China (Grant no. 31372050) and the Natural Science Foundation of Shandong Province (Grant no. ZR2014CM015).

\section{ACKNOWLEDGMENT}

We thank all co-authors who participated in the studies mentioned in the text that were published by our groups.

mosaic virus, in Arabidopsis. Biochem. Biophys. Res. Commun. 477, 626-632. doi: 10.1016/j.bbrc.2016.06.110

Hosoda, K., Imamura, A., Katoh, E., Hatta, T., Tachiki, M., Yamada, H., et al. (2002). Molecular structure of the GARP family of plant Myb-related DNA binding motifs of the Arabidopsis response regulators. Plant Cell 14, 2015-2029. doi: $10.1105 /$ tpc.002733

Jenkins, M. T. (1926). A second gene producing golden plant color in maize. Am. Nat. 60, 484-488. doi: 10.1086/280119

Jhadeswar, M., Michael, W., Ghislaine, A., Radhey, P., Darrell, D., Jas, S., et al. (2014). Arabidopsis GOLDEN2-LIKE (GLK) transcription factors activate jasmonic acid (JA)-dependent disease susceptibility to the biotrophic pathogen Hyaloperonospora arabidopsidis, as well as JA-independent plant immunity against the necrotrophic pathogen Botrytis cinerea. Mol. Plant Pathol. 15, 174-184. doi: 10.1111/mpp.12077

Jones, B., Frasse, P., Olmos, E., Zegzouti, H., Li, Z. G., Latché, A., et al. (2002) Down-regulation of DR12, an auxin-response-factor homolog, in the tomato results in a pleiotropic phenotype including dark green and blotchy ripening fruit. Plant J. 32, 603-613. doi: 10.1046/j.1365-313X.2002.01450.x

Kim, J. H., Woo, H. R., Kim, J., Lim, P. O., Lee, I. C., Choi, S. H., et al. (2009). Trifurcate feed-forward regulation of age-dependent cell death involving miR164 in Arabidopsis. Science 323, 1053-1057. doi: 10.1126/science.1166386

Kobayashi, K., Baba, S., Obayashi, T., Sato, M., Toyooka, K., Keränen, M., et al. (2012). Regulation of root greening by light and auxin/cytokinin signaling in Arabidopsis. Plant Cell 24, 1081-1095. doi: 10.1105/tpc.111.092254

Kobayashi, K., Fujii, S., Sasaki, D., Baba, S., Ohta, H., Masuda, T., et al. (2014). Transcriptional regulation of thylakoid galactolipid biosynthesis coordinated with chlorophyll biosynthesis during the development of chloroplast in Arabidopsis. Front. Plant Sci. 5:272. doi: 10.3389/fpls.2014.00272

Kobayashi, K., Narise, T., Sonoike, K., Hashimoto, H., Sato, N., Kondo, M., et al. (2013). Role of galactolipid biosynthesis in coordinated development 
of photosynthetic complexes and thylakoid membranes during chloroplast biogenesis in Arabidopsis. Plant J. 73, 250-261. doi: 10.1111/tpj.12028

Kunkel, B. N., and Brooks, D. M. (2002). Cross talk between signaling pathways in pathogen defense. Curr. Opin. Plant Biol. 5, 325-331. doi: 10.1016/S13695266(02)00275-3

Langdale, J. A., and Kidner, C. A. (1994). Bundle sheath defective, a mutation that disrupts cellular differentiation in maize leaves. Development 120, 673-681.

Lawton, K. A., Weymann, K., Friedrich, L., Vernooij, B., Uknes, S., and Ryals, J. (1995). Systemic acquired resistance in Arabidopsis requires salicylic acid but not ethylene. Mol. Plant Microbe Interact. 8, 863-870. doi: 10.1094/MPMI-80863

Leister, D., and Kleine, T. (2016). Definition of a core module for the nuclear retrograde response to altered organellar gene expression identifies GLK overexpressors as gun mutants. Physiol. Plant. 157, 297-309. doi: 10.1111/ ppl.12431

Li, X., Clarke, J. D., Zhang, Y., and Dong, X. (2001). Activation of an EDS1mediated R-gene pathway in the sncl mutant leads to constitutive, NPR1independent pathogen resistance. Mol. Plant Microbe Interact. 14, 1131-1139. doi: 10.1094/MPMI.2001.14.10.1131

Massoud, K., Barchietto, T., Le Rudulier, T., Pallandre, L., Didierlaurent, L., Garmier, M., et al. (2012). Dissecting phosphite-induced priming in Arabidopsis infected with Hyaloperonospora arabidopsidis. Plant Physiol. 159, 286-298. doi: 10.1104/pp.112.194647

Murray, S. L., Thomson, C., Chini, A., Read, N. D., and Loake, G. J. (2002). Characterization of a novel, defense-related Arabidopsis mutant, cir1, isolated by luciferase imaging. Mol. Plant Microbe Interact. 15, 557-566. doi: 10.1094/MPMI.2002.15.6.557

Nadakuduti, S. S., Holdsworth, W. L., Klein, C. L., and Barry, C. S. (2014). KNOX genes influence a gradient of fruit chloroplast development through regulation of GOLDEN2-LIKE expression in tomato. Plant J. 78, 1022-1033. doi: $10.1111 /$ tpj.12529

Nagatoshi, Y., Mitsuda, N., Hayashi, M., Inoue, S. I., Okuma, E., Kubo, A., et al. (2016). GOLDEN2-LIKE transcription factors for chloroplast development affect ozone tolerance through the regulation of stomatal movement. Proc. Natl. Acad. Sci. U.S.A. 113, 4218-4223. doi: 10.1073/pnas.1513093113

Nakamura, H., Muramatsu, M., Hakata, M., Ueno, O., Nagamura, Y., Hirochika, H., et al. (2009). Ectopic overexpression of the transcription factor OsGLK1 induces chloroplast development in non-green rice cells. Plant Cell Physiol. 50, 1933-1949. doi: 10.1093/pcp/pcp138

Nguyen, C. V., Vrebalov, J. T., Gapper, N. E., Zheng, Y., Zhong, S., Fei, Z., et al. (2014). Tomato GOLDEN2-LIKE transcription factors reveal molecular gradients that function during fruit development and ripening. Plant Cell 26, 585-601. doi: 10.1105/tpc.113.118794

Oh, E., Zhu, J. Y., and Wang, Z. Y. (2012). Interaction between BZR1 and PIF4 integrates brassinosteroid and environmental responses. Nat. Cell Biol. 14, 802-809. doi: $10.1038 / \mathrm{ncb} 2545$

Pedro, R., José Luis, M., and Víctor, Q. (2012). Arabidopsis MDA1, a nuclearencoded protein, functions in chloroplast development and abiotic stress responses. PLoS ONE 7:e42924. doi: 10.1371/journal.pone.0042924

Powell, A. L., Nguyen, C. V., Hill, T., Cheng, K. L., Figueroabalderas, R., Aktas, H., et al. (2012). Uniform ripening encodes a golden 2-like transcription factor regulating tomato fruit chloroplast development. Science 336, 1711-1715. doi: $10.1126 /$ science. 1222218

Rairdan, G. J., and Delaney, T. P. (2002). Role of salicylic acid and NIM1/NPR1 in race-specific resistance in Arabidopsis. Genetics 161, 803-811.

Rauf, M., Arif, M., Dortay, H., Matallana-Ramírez, L. P., Waters, M. T., Gil Nam, H., et al. (2013). ORE1 balances leaf senescence against maintenance by antagonizing G2-like-mediated transcription. EMBO Rep. 14, 382-388. doi: 10.1038/embor.2013.24

Rensing, S. A., Daniel, L., Zimmer, A. D., Astrid, T., Asaf, S., Harris, S., et al. (2008). The physcomitrella genome reveals evolutionary insights into the conquest of land by plants. Science 319, 64-69. doi: 10.1126/science.11 50646
Riechmann, J., Heard, J., Martin, G., Reuber, L., Jiang, C., Keddie, J., et al. (2000). Arabidopsis transcription factors: genome-wide comparative analysis among eukaryotes. Science 290, 2105-2110. doi: 10.1126/science.290.5499.2105

Rossini, L., Cribb, L., Martin, D. J., and Langdale, J. A. (2001). The maize Golden2 gene defines a novel class of transcriptional regulatorsin plants. Plant Cell 13, 1231-1244. doi: 10.1105/tpc.13.5.1231

Ryals, J. A., Neuenschwander, U. H., Willits, M. G., Molina, A., Steiner, H. Y., and Hunt, M. D. (1996). Systemic acquired resistance. Plant Cell 10, 1809-1819. doi: 10.1105/tpc.8.10.1809

Sagar, M., Chervin, C., Mila, I., Hao, Y. W., Roustan, J. P., Benichou, M., et al. (2013). SlARF4, an auxin response factor involved in the control of sugar metabolism during tomato fruit development. Plant Physiol. 161, 1362-1374. doi: 10.1104/pp.113.213843

Savitch, L. V., Ghislaine, A., Motoaki, S., Robert, L. S., Tinker, N. A., Huner, N. P. A., et al. (2005). The effect of overexpression of two brassica CBF/DREB1like transcription factors on photosynthetic capacity and freezing tolerance in Brassica napus. Plant Cell Physiol. 46, 1525-1539. doi: 10.1093/pcp/pci165

Savitch, L. V., Subramaniam, R., Allard, G. C., and Singh, J. (2007). The GLK1 'regulon' encodes disease defense related proteins and confers resistance to Fusarium graminearum, in Arabidopsis. Cereal Res. Commun. 359, 234-238. doi: 10.1016/j.bbrc.2007.05.084

Song, Y., Yang, C., Gao, S., Zhang, W., Li, L., and Kuai, B. (2014). Age-triggered and dark-induced leaf senescence require the bHLH transcription factors PIF3, 4, and 5. Mol. Plant 7, 1776-1787. doi: 10.1093/mp/ssul09

Tamai, H., Iwabuchi, M., and Meshi, T. (2002). Arabidopsis GARP transcriptional activators interact with the pro-rich activation domain shared by G-boxbinding bZIP factors. Plant Cell Physiol. 43, 99-107. doi: 10.1093/pcp/ pcf011

Tang, X., Miao, M., Niu, X., Zhang, D., Cao, X., Jin, X., et al. (2015). Ubiquitinconjugated degradation of golden 2-like transcription factor is mediated by CUL4-DDB1-based E3 ligase complex in tomato. New Phytol. 209, 1028-1039. doi: $10.1111 / \mathrm{nph} .13635$

Tomko, R. J., and Hochstrasser, M. (2012). Molecular architecture and assembly of the eukaryotic proteasome. Annu. Rev. Biochem. 82, 415-445. doi: 10.1146/annurev-biochem-060410-150257

Wang, P., Fouracre, J., Kelly, S., Karki, S., Gowik, U., Aubry, S., et al. (2013). Evolution of GOLDEN2-LIKE gene function in C3 and C4 plants. Planta 237, 481-495. doi: 10.1007/s00425-012-1754-3

Waters, M. T., Moylan, E. C., and Langdale, J. A. (2008). GLK transcription factors regulate chloroplast development in a cell-autonomous manner. Plant J. 56, 432-444. doi: 10.1111/j.1365-313X

Waters, M. T., Wang, P., Korkaric, M., Capper, R. G., Saunders, N. J., and Langdale, J. A. (2009). GLK transcription factors coordinate expression of the photosynthetic apparatus in Arabidopsis. Plant Cell 21, 1109-1128. doi: $10.1105 /$ tpc. 108.065250

Yasumura, Y., Moylan, E., and Langdale, J. A. (2005). A conserved transcription factor mediates nuclear control of organelle biogenesis in anciently diverged land plants. Plant Cell 17, 1894-1907. doi: 10.1105/tpc.105.033191

Yu, X., Li, L., Zola, J., Aluru, M., Ye, H., Foudree, A., et al. (2011). A brassinosteroid transcriptional network revealed by genome-wide identification of BESI target genes in Arabidopsis thaliana. Plant J. 65, 634-646. doi: 10.1111/j.1365313X.2010.04449

Conflict of Interest Statement: The authors declare that the research was conducted in the absence of any commercial or financial relationships that could be construed as a potential conflict of interest.

Copyright (c) 2016 Chen, Ji, Wen, Liu, Li, Chen, Gao and Li. This is an open-access article distributed under the terms of the Creative Commons Attribution License (CC BY). The use, distribution or reproduction in other forums is permitted, provided the original author(s) or licensor are credited and that the original publication in this journal is cited, in accordance with accepted academic practice. No use, distribution or reproduction is permitted which does not comply with these terms. 